\title{
DICHOTOMIES FOR SYSTEMS WITH DISCONTINUOUS COEFFICIENTS
}

\author{
RAúl NaUlin
}

\begin{abstract}
In this work we are concerned with the problem of the existence of an exponential dichotomy for the linear singularly perturbed system $\varepsilon x^{\prime}=A(t) x$, where the matrix $A(t)$ is piecewise uniformly continuous, that is, $A(t)$ admits points of discontinuity but is uniformly continuous in any interval where it is continuous. We shall prove that the classical result regarding the existence of an exponential dichotomy extends to this case, when there is a constant $\gamma>0$ such that $|\operatorname{Re} \lambda(t)| \geqslant$ $\gamma>0$ for any eigenvalue $\lambda(t)$ of $A(t)$. The proofs are obtained by means of the quasidiagonalisation of a non-constant matrix: For $A(t)$, a piecewise uniformly continuous matrix and $\sigma>0$ there exists a bounded, piecewise constant function $L(t): J \rightarrow \mathbb{C}^{n \times n}$, and a bounded matrix $\Delta(t, \sigma)$ such that $L^{-1}(t) A(t) L(t)=$ $\Lambda(t)+\Delta(t, \sigma),|\Delta(t, \sigma)| \leqslant \sigma$, where $\Lambda(t)$ is the diagonal matrix consisting of eigenvalues of $A(t)$.
\end{abstract}

\section{INTRODUCTION}

In our work we are concerned with the existence of an exponential dichotomy for the singularly perturbed system

$$
\varepsilon x^{\prime}=A(t) x \text {. }
$$

By this, we mean the existence of a fundamental matrix $\Phi$ of (1), constants $K \geqslant 1$ and $\alpha>0$ not depending on $\varepsilon$, a bound $\varepsilon_{0}$, and a projection matrix $P(P P=P)$ such that the estimates:

$$
\left|\Phi(t) P \Phi^{-1}(s)\right|,\left|\Phi(s)(I-P) \Phi^{-1}(t)\right| \leqslant K e^{-\alpha(t-s) / e}
$$

are valid for $t \geqslant s$ and $\varepsilon \in\left(0, \varepsilon_{0}\right]$.

For the "regular" system $x^{\prime}=A(t) x$ this problem has been intensively studied. We refer in this case to the book [4].

Received 12 February 1991

The author wishes to express his gratitude to Professor Margaret $R$. Bledsoe for her revision of the English version of this article.

Copyright Clearance Centre, Inc. Serial-fee code: 0004-9729/92 \$A2.00+0.00. 
The linear singularly perturbed system (1) presents many interesting features. For example if we use the fast scale $s=\varepsilon^{-1}\left(t-t_{0}\right), t \geqslant t_{0}$, then (1) takes the form $(\dot{y}=d y / d s)$

$$
\dot{y}=A\left(\varepsilon s+t_{0}\right) y \text {. }
$$

Formally putting $\varepsilon=0$ in (3) we may "freeze" the time to obtain the system

$$
\dot{y}=A\left(t_{0}\right) y
$$

The system (4) does not depend on $\varepsilon$. The function $e^{A\left(t_{0}\right) \cdot}$ is a fundamental matrix of (4) and it is shown in [5] that for $t \geqslant t_{0}$ the function $e^{A\left(t_{0}\right)(t-\alpha) / \varepsilon}$ gives for small $\varepsilon$, an excellent approximation of the fundamental matrix $X(t)(X(0)=I)$ of system (1). No similar treatment is possible for the regular case. Thus, it seems that the problem of the existence of an exponential dichotomy for the singularly perturbed case allows a special approach. We will show that the continuity of $A(t)$, a condition which is generally required in the theory of exponential dichotomies, can in this case be removed, so that points of discontinuities may be allowed in the function $A(t)$. In what follows $A(t)$ is a bounded, piecewise uniformly continuous, (see below for the definition of a piecewise uniformly continuous function) $n \times n$ matrix function of $t$ with domain $J=[0,1]$, $J=(-\infty, \infty)$ or $J=[0, \infty)$. In this paper we prove results only for $J=\mathbb{R}$, in order to simplify the presentation. Such discontinuous matrices have been considered in the papers $[9,7]$ concerning new formulations of the shadowing lemma. The classical proof of the theorem regarding the existence of an exponential dichotomy, as given in [4], consists of three steps: $A$ transformation of the matrix $A(t)$ into a block diagonal matrix $D$, the existence of exponential dichotomy for the system $\varepsilon y^{\prime}=D y$ and the use of the roughness theorem. The second step is accomplished by obtaining an estimate of the exponential matrix $e^{D(t) s}$ for $s \geqslant 0$. In our treatment we avoid this estimate. Instead, we rely on ideas presented in [8], where the quasidiagonalisation of a time varying matrix function is accomplished by means of a piecewise continuous function: $L^{-1}(t) A(t) L(t)=\Lambda(t)+\Delta(t)$, where $\Lambda(t)$ is a diagonal matrix and $\Delta$ is small. Our work is a further development of this idea.

The existence of exponential dichotomies has been recognised as a fact of great importance not only from a theoretical point of view. For example in [1] this concept is applied to the boundary value problem $\varepsilon x^{\prime}=A(t) x+f(t), B_{a} y(a)+B_{b} y(b)=B$, $t \in[a, b]$, and a close relation is established between the existence of an exponential dichotomy for the homogeneous linear system and the well conditioning of boundary value problems. 


\section{DEFINITIONS AND BASIC FACTS}

In what follows $|x|$ is an arbitrary norm on $\mathbb{C}$, and $|A|$ is the respective operator norm. Throughout this paper $P$ and $Q$ denote the following orthogonal projections

$$
P=\left[\begin{array}{ccc}
I_{k} & \vdots & 0 \\
\ldots & \ldots & \ldots \\
0 & \vdots & 0
\end{array}\right], \quad Q=I-P
$$

where $I_{k}$ is the $k \times k$ unit matrix.

Definition 1: The function $A: J \rightarrow \mathbb{C}^{n \times n}$ is called piecewise uniformly continuous if and only if the following hold:

(1) $A$ has a countable set $\mathcal{D}=\left\{\ldots t_{i}, t_{i+1}, t_{i+2}, \ldots\right\}$ of discontinuities $\left(t_{i}<t_{j}\right.$ if $\left.i<j\right)$.

(2) There exists $\mu>0$ such that $\left|t_{i}-t_{j}\right| \geqslant \mu$ for $t_{i} \neq t_{j}, t_{i}, t_{j} \in \mathcal{D}$.

(3) Given $\varepsilon>0$, there exists $\delta(\varepsilon)>0$ such that for all $t_{i} \in \mathcal{D}, s, t \in$ $\left(t_{i}, t_{i+1}\right)$, and $|s-t|<\delta(\varepsilon)$ implies that $|A(t)-A(s)|<\varepsilon$.

Definition 2: We say that the complex number $u$ is less than the complex number $v$ if and only if $\operatorname{Re} u<\operatorname{Re} v$ or $\operatorname{Re} u=\operatorname{Re} v$ and $\operatorname{Im} u<\operatorname{Im} v$.

To denote that $u$ is less than $v$ we write $u<v$.

Definition 3: We call the diagonal matrix $\Lambda=\operatorname{diag}\left\{\lambda_{1}, \lambda_{2}, \ldots, \lambda_{n}\right\}$ lexicographically ordered if and only if $i<j$ implies $\lambda_{i}<\lambda_{j}$ or $\lambda_{i}=\lambda_{j}$.

In the following we require the next result [2]:

THEOREM 4. (quasidiagonalisation of a constant matrix). Let $A$ be a constant matrix with complex elements. Then for any $\sigma>0$ there exists a non-singular matrix $Q$, depending on $\sigma$, such that $Q^{-1} A Q=\Lambda+\Gamma$, where $\Lambda=\operatorname{diag}\left\{\lambda_{1}, \lambda_{2}, \ldots, \lambda_{n}\right\}$ is the diagonal matrix of eigenvalues of $A$, lexicographically ordered, and $|\Gamma| \leqslant \sigma$.

\section{QUASIDIAGONALISATION OF NON-CONSTANT MATRICES}

We begin this section with a technical lemma:

LEMma 5. Let $\left\{I_{i}, i \in \mathcal{L}\right\}$ be a collection of finite closed intervals such that for a fixed number $\sigma$ one has $\ell\left(I_{i}\right) \geqslant \sigma(\ell(I)$ is the length of the interval $I)$ for all $i \in \mathcal{L}$. Then, given $h$ with $0<h<\sigma$, for any $I_{i}$ there exists a number $a_{i}$, a number $h_{i}$, and an integer $k_{i}$ such that $h / 2 \leqslant h_{i} \leqslant h, I_{i}=\left[s_{0}, s_{1}\right] \cup\left[s_{1}, s_{2}\right] \cup \ldots \cup\left[s_{k_{i}-1}, s_{k_{i}}\right]$ and $s_{i}=a_{i}+i h_{i}$ for $i=0,1, \ldots, k_{i}$.

Proof: Let $I_{i}=\left[a_{i}, b_{i}\right], l\left(I_{i}\right)=k_{i}(h / 2)+\theta_{i}, 0 \leqslant \theta_{i}<h / 2$. Then $l\left(I_{i}\right)=$ $k_{i}\left((h / 2)+\theta_{i} / k_{i}\right)$. Let us define $h_{i}:=h / 2+\theta_{i} / k_{i}$ and $s_{i}=a_{i}+i h_{i}, i=0,1, \ldots, k_{i}$. 
THEOREM 6. If $A(t)$ is piecewise uniformly continuous, $|A(t)| \leqslant M$ on $J$, then for a number $\sigma>0$ there exists an $h(\sigma)>0$, a countable set $\mathcal{U}=\left\{\ldots, s_{i}, s_{i+1}, s_{i+2} \ldots\right\}$ $\subset \mathbb{R}$ and a finite collection $\tau$ of non-singular matrices such that:

(1) $\mathcal{D}$, the set of discontinuities of $A$, is contained in $U$;

(2) $h / 2 \leqslant s_{i+1}-s_{i} \leqslant h$;

(3) for any interval $\left(s_{i}, s_{i+1}\right)$ there exists $L \in \tau$ such that $L A(t) L^{-1}=$ $\Lambda(t)+\Delta(t, \sigma)$, where $\Lambda(t)$ is the diagonal matrix of eigenvalues of $A(t)$, lexicographically ordered, and $|\Delta(t, \sigma)| \leqslant \sigma$, for all $t \in\left(s, s_{i+1}\right)$.

Proof: Let us denote by $\mathcal{B}$ the set of $n \times n$ square matrices $C$ such that $|C| \leqslant M$. $\mathcal{B}$ is a compact subset of $\mathbb{C}^{n \times n}$. Let $\sigma>0$ be given. Then, according to Theorem 4 , for any $C \in \mathcal{B}$ we can define a non-singular matrix $L$ such that $L^{-1} C L=\Lambda_{C}+\Delta$, where $\Lambda_{C}$ is the diagonal matrix with the eigenvalues of $C$, lexicographically ordered, and $\Delta$ is such that $|\Delta| \leqslant \sigma / 2$. Therefore for $D \in \mathcal{B}$ we can write: $L^{-1} D L=\Lambda_{D}+\Lambda_{C}-\Lambda_{D}+$ $L^{-1}(D-C) L+\Delta$. This last identity shows, by virtue of the continuity of the eigenvalue functions, that we can fix a real number $r(\sigma)>0$ such that $L^{-1} C L=\Lambda_{D}+\Delta$, with $|\Delta| \leqslant \sigma$ if $|C-D| \leqslant r(\sigma)$. This means that every matrix $D$ in the closed ball $B[C, r(\sigma)]$ is $\sigma$-quasi diagonalisable by the same non-singular matrix $L$ as in $C$. Now using the compactness of $\mathcal{B}$ we can define a finite collection of matrices $C_{j}$, nonsingular matrices $L_{j}$, and radii $r_{j}, j=1,2, \ldots$, such that $\mathcal{B}=\cup_{j=1}^{k}\left(B\left[C_{j}, r_{j}\right] \cap \mathcal{B}\right)$ and $L_{j}^{-1} C L_{j}=\Lambda_{D}+\Delta$, with $|\Delta| \leqslant \sigma$ for any $D \in B\left[C_{j}, r_{j}\right]$. We will denote by $\xi$ the Lebesgue number of this covering, that is, for any subset $\mathcal{A} \subseteq \mathcal{B}$ with diameter $d(\mathcal{A})=$ Sup $\{|A-B|: A, B \in \mathcal{A}\} \leqslant \xi$, there exists a ball $B\left[C_{j}, r_{j}\right]$ containing $\mathcal{A}$. Finally we let $\tau=\left\{L_{1}, L_{2}, \ldots L_{k}\right\}$. Let $\mathcal{D}=\left\{\ldots t_{i}, t_{i+1}, t_{i+2}, \ldots\right\}$ be the set of discontinuities of $A(t)$ and $\mu>0$ the number which appears in the definition of the piecewise uniformly continuous function $A(t)$. Let $h$ be a number such that $|t-s|<h<\mu$ implies $|A(t)-A(s)| \leqslant \xi$ for $s, t \in\left(t_{i}, t_{i+1}\right)$ an interval of continuity of $A(t)$. Now, if $A(t)$ is continuous on $\left(-\infty, t_{j}\right)$ then we define the numbers $s_{i}=t_{j}-i(h / 2), i=0,1,2 \ldots$, and we form the set $\mathcal{U}$ by adding these points to the set $\mathcal{D}$. We define an analogous collection of points if $A(t)$ is continuous on the interval $\left(t_{i}, \infty\right)$ and add these to the set $\mathcal{U}$ as well. For the set of finite intervals $\left[t_{i}, t_{i+1}\right]$ we add to $\mathcal{U}$ all the partitions of these intervals indicated in Lemma 5.

The above theorem proves that there exists a function $L: J / U \rightarrow \mathbb{C}^{n \times n}$ which is constant on $\left(s_{i}, s_{i+1}\right)$ such that for all $t \in\left(s_{i}, s_{i+1}\right), s_{i}, s_{i+1} \in \mathcal{U}, L^{-1}(t) A(t) L(t)=$ $\Lambda(t)+\Delta(t),|\Delta(t)| \leqslant \sigma$, with $\sigma$ small.

\section{EXPONENTIAL DiCHOTOMIES FOR A LINEAR SINGULARLY PERTURBED SYSTEM WITH NO DISCONTINUOUS COEFFICIENTS}

Let us consider the singularly perturbed system (1) under the following conditions: 
(C1) $A(t): \mathbb{R} \rightarrow \mathbb{C}^{n \times n}$ is a piecewise uniformly continuous function and $|A(t)| \leqslant M$, for all $t \in \mathbb{R}$.

(C2) There exists an integer $k, 0 \leqslant k \leqslant n$, such that $k$ eigenvalues of $A(t)$ satisfy $\operatorname{Re} \lambda(t) \leqslant-\gamma$, and $n-k$ eigenvalues of $A(t)$ satisfy $\operatorname{Re} \lambda(t) \geqslant$ $\gamma>0$, where $\gamma$ is a constant.

(C3) $A(t)$ is almost everywhere differentiable on $J$, and $\left|A^{\prime}(t)\right|$ is bounded on $J$.

We will consider the solutions of the system $\varepsilon x^{\prime}=A(t) x$ in a generalised sense, that is, the solutions of this linear system must be absolutely continuous functions [6]. The following theorem can be proven just as in the case when $A$ is a $C^{1}$ function (see the proof of Proposition 1 of Chapter 6 in [4]).

Theorem 7. Let $A(t)$ satisfy (C1), (C2) and (C3). Then there exists an absolutely continuous function matrix $T(t)$ such that:

(1) $T^{-1}(t)$ exists for all $t \in \mathbb{R}$;

(2) $\left|T^{-1}(t)\right||T(t)| \leqslant M_{1}$;

(3) $\left|T^{-1}(t) T^{\prime}(t)\right| \leqslant M_{2}$;

(4) the change of variable $x=T(t) y$ reduces the system (1) to

$$
\varepsilon y^{\prime}=\left[D(t)-\varepsilon T^{-1}(t) T^{\prime}(t)\right] y
$$

where $D(t)=\operatorname{diag}\left(D_{1}(t), D_{2}(t)\right)$, all the eigenvalues of $D_{1}(t)$ have negative real parts, and all eigenvalues of $D_{2}(t)$ have positive real parts.

Given the system (6) we will prove that the system

$$
\varepsilon z^{\prime}=D(t) z
$$

has an exponential dichotomy. We remark that (7) can be written as a decoupled system

$$
\begin{aligned}
& \varepsilon u^{\prime}=D_{1}(t) u \\
& \varepsilon v^{\prime}=D_{2}(t) v .
\end{aligned}
$$

We denote by $\Phi_{1}$ and $\Phi_{2}$ the fundamental matrices of systems (8) and (9), with the initial condition $\Phi_{1}(0)=I_{k}, \Phi_{2}(0)=I_{n-k}$.

TheOREM 8. Let $A(t)$ satisfy the conditions of Theorem 7. Then there exist an $\varepsilon_{0}>0$, and a constant $H \geqslant 1$ such that:

$$
\left|\Phi_{1}(t) \Phi_{1}^{-1}(s)\right|,\left|\Phi_{2}(s) \Phi_{2}^{-1}(t)\right| \leqslant H e^{-\gamma(t-s) / 4 e}, \quad t \geqslant s .
$$

Proof: We shall prove this only for the system (8). According to Theorem 6 we define a function $L(t)$, which is equal to a constant matrix $L_{i+1}$ on each interval 
$\left[s_{i}, s_{i+1}\right], s_{i}, s_{i+1} \in \mathcal{U}$, where it quasidiagonalises the matrix $D_{1}(t)$. Let $s<t$, $s \leqslant s_{0}<s_{1}<\ldots<s_{m} \leqslant t, s_{0}, s_{1}, \ldots s_{m} \in \mathcal{U}$.

On the interval $\left[s, s_{0}\right]$ we make the change of variable $u=L_{0} y$, and the system (8) takes the form

$$
\varepsilon y^{\prime}=(\Lambda(t)+\Delta(t, \sigma)) y .
$$

In this case, the matrix solution $Y(t)$ of this system with the condition $Y(s)=I$ satisfies: $\left|Y\left(s_{0}\right) Y^{-1}(s)\right| \leqslant e^{-(\gamma-\sigma)\left(s_{0}-s\right) / \varepsilon}$. This means that for the matrix solution $\Phi$ of (8) with $\Phi(s)=I$ we have the estimate

$$
\left|\Phi\left(s_{0}\right) \Phi^{-1}(s)\right| \leqslant\left|L_{0}\right|\left|L_{1}^{-1}\right| e^{-(\gamma-\sigma)\left(s_{0}-s\right) / \varepsilon} .
$$

Taking into account that the range of the function $L(t)$ is finite, we can determine a number $K$ such that $|L(t)|\left|L^{-1}(t)\right| \leqslant K$ for any $t$. So we have

$$
\left|\Phi\left(s_{0}\right) \Phi^{-1}(s)\right| \leqslant K e^{-(\gamma-\sigma)\left(s_{0}-s\right) / e} .
$$

Let us make the change of variable $u=L_{1} y$ in the system (7) on the interval $\left[s_{0}, s_{1}\right]$. Again we obtain a system of the form (9), and we solve it with the initial condition $Y\left(s_{0}\right)=L_{1}^{-1} \Phi\left(s_{0}\right) \Phi^{-1}(s)$. In this case we obtain the estimate $\left|Y\left(s_{2}\right) Y^{-1}\left(s_{0}\right)\right| \leqslant$ $e^{-(\gamma-\sigma)\left(s_{1}-s_{0}\right) / \varepsilon}$. For the matrix solution $\Phi$ of system (8), with initial condition $\Phi\left(s_{0}\right) \Phi^{-1}(s)$ we have the estimate:

$$
\left|\Phi\left(s_{1}\right) \Phi^{-1}\left(s_{0}\right)\right| \leqslant\left|L_{1}\right|\left|L_{1}^{-1}\right| e^{-(\gamma-\sigma)\left(s_{1}-s_{0}\right) / \varepsilon} \leqslant K e^{-(\gamma-\sigma)\left(s_{1}-s_{0}\right) / \varepsilon} .
$$

From these estimates we obtain $\left|\Phi\left(s_{1}\right) \Phi^{-1}(s)\right| \leqslant K^{2} e^{-(\gamma-\sigma)\left(s_{1}-s\right) / e}$. Repeating this argument we find that

$$
\left|\Phi(t) \Phi^{-1}(s)\right| \leqslant K^{m+2} e^{-(\gamma-\sigma)(t-s) / \epsilon} .
$$

Now we observe that

$$
\begin{aligned}
K^{m+2} & =K^{2} \exp \{m \operatorname{Ln} K\}=K^{2} \exp \left\{m(h / 2)(h / 2)^{-1} \operatorname{Ln} K\right\} \\
& \leqslant K^{2} \exp \left\{\left(s_{m}-s_{0}\right)(2 / h) \operatorname{Ln} K\right\} \leqslant K^{2} \exp \{(t-s)(2 / h) \operatorname{Ln} K\} .
\end{aligned}
$$

From this it follows that $\left|\Phi(t) \Phi^{-1}(s)\right| \leqslant K^{2} e^{-(\gamma-\sigma+e(2 / h) \operatorname{Ln} K)(t-s) / e p}$.

This last inequality shows that if $\sigma \leqslant \gamma / 4$, then for $\varepsilon_{0}(2 / h) \operatorname{Ln} K \leqslant \gamma / 4,0<\varepsilon \leqslant \varepsilon_{0}$ and $H=K^{2}$, we obtain:

$$
\left|\Phi(t) \Phi^{-1}(s)\right| \leqslant H^{2} e^{-\gamma(t-s) / 4 \epsilon}, t \geqslant s .
$$

Finally we note that (11) does not depend upon the fact that $\Phi(s)=I$. 
Note 1. Essentially, for a bounded, uniformly continuous coefficient matrix $A(t)$ this theorem is proved in [4].

Note 2. The above proof shows that the constants $M, \varepsilon_{0}$ are independent of the jumps in the function $A(t)$ at the points of discontinuity.

From Theorem 8 we obtain, relying on the roughness theorem for an exponential dichotomy (see [4], Chapter 5), the following

THEOREM 9. If $4 \varepsilon_{0} M_{2} \leqslant \gamma / 36 K^{5}$ then the system (6) has the exponential dichotomy:

$$
\left|X(t) P X^{-1}(s)\right|,\left|X(s) Q X^{-1}(t)\right| \leqslant 12 H^{3} \exp \left\{-\left(\gamma-48 H^{3} M_{2} \varepsilon_{0}\right)(t-s) / 4 \varepsilon\right\}
$$

for $0<\varepsilon \leqslant \varepsilon_{0}$ and $t \geqslant s$.

From the second estimate given in Theorem 7, it follows that the existence of an exponential dichotomy for (6) implies the existence of an exponential dichotomy for the system (1).

\section{REFERENCES}

[1] U.M. Ascher, R.M.M. Matheij and R.D. Russel, Numerical solution of boundary value problems for ordinary differential equations (Prentice Hall, New Jersey, 1988).

[2] R. Bellman, Stability theory of differential equations (Dover Publications, New York, 1953).

[3] K.W. Chang, 'Almost periodic solutions of singularly perturbed systems of differential equations', J. Differential Equations 4 (1968), 300-307.

[4] W.A. Coppel, Dichotomies in stability theory: Lecture notes in mathematics 629 (Springer Verlag, Berlin, 1978).

[5] S.H. Javid, 'Uniform asymptotic stability of linear time varying singularly perturbed systems', J. Franklin Inst. 305 (1978), 27-37.

[6] E.A. Coddington and N. Levinson, Theory of ordinary differential equations (McGraw-Hill, New York, 1955).

[7] X.B. Lin, 'Shadowing lemma and singularly perturbed boundary value problems', SIAM J. Appl. Math. 49 (1989), 26-54.

[8] V.I. Rožkov, 'Periodic solutions of linear systems with a small parameter in the derivative', Soviet Math. Dokl. 16 (1975).

[9] K.J. Palmer, 'Exponential dichotomies and transversal homoclinic points', J. Differential Equations 55 (1984), 225-256. 